\title{
IMPLEMENTATION OF NEWPRED AND UEP FOR VIDEO QUALITY IMPROVEMENTS FOR MPEG-4 OVER Simulated UMTS CHANNEL PROPAgATION ENVIRONMENTS
}

\author{
Dr. Bhumin H. Pathak ${ }^{1}$, Dr. Geoff Childs ${ }^{2}$ and Dr. Maaruf Ali ${ }^{3}$ \\ ${ }^{1}$ Airvana Inc., Chelmsford, USA \\ bhumin.pathak@gmail.com \\ ${ }^{2}$ School of Technology at Oxford Brooks University, Oxford, UK \\ gnchilds@brookes.ac.uk \\ ${ }^{3}$ School of Technology at Oxford Brooks University, Oxford, UK \\ mali@brookes.ac.uk
}

\begin{abstract}
Received video quality enhancement gained with the implementation of NEWPRED and UEP mechanism is discussed in this paper. Selection of these two methods is justified with details of their implementation. Several MPEG-4 video test bit-streams are transmitted over simulated UMTS environment. Performance enhancement over various channel propagation conditions as standardized by 3GPP for conformance testing in is quantified. Objective video quality assessment is done using standard PSNR metric while near subjective assessment is obtained using Video Quality Metric (VQM) utility. It is observed that in all propagation conditions simulated, implementation of proposed error concealment and mitigation techniques provide considerable improvements in both subjective and objective video quality.
\end{abstract}

\section{KEYWORDS}

NEWPRED, MPEG-4, UMTS, UEP

\section{INTRODUCTION}

As bandwidth is a limited and valuable resource in wireless environment, video compression standards play important role for real time video transmission services. Typical video compression process exploits redundancies in the raw video from different domains and tries to compress information energy in as few bits as possible and saves the bandwidth required for transmission. This process has unfortunate trade off between compression achieved and sensitivity to transmission errors. Loss of few bits during transmission over wireless channel can result in high information loss and it results into poor received video quality. Different wireless communication standards such as UMTS provide some degree of error protection and other QoS (Quality of Services) related services but additional mechanisms are still required to provide higher degree of end user service experience.

As discussed above, video compression process exploits data redundancies from all domains [1]. One such domain is redundancy between consecutive frames which is known as inter-frame redundancy. High degree of compression is achieved by inter-frame compression technique. Different levels of hierarchical structures are used to maximize compression advantage that can be gained from this process but on other hand transmission error 
damaging some crucial part of this hierarchical structure can result into high degree of error propagation into video stream. This research primarily concentrates on minimizing error propagation within the video stream.

This problem of error propagation is identified and analyzed in the proposed research. Errors are categorized according to their severity. Two different methods are proposed to deal with propagation errors. One of the methods utilizes the automatic repeat request (ARQ) mechanism used by UMTS architecture by providing Unequal Error Protection (UEP) while the other exploits dynamic frame referencing technique proposed in MPEG-4 video compression standard known as NEWPRED. These two methods effectively conceal error propagation further into video stream and their implementation results into improved received video quality at significant degree.

This improved video quality comes at the cost of some processing and transmission overheads. Implementation complexities and transmission overheads involved with two proposed methods are compared with those of classical forward error correction mechanism. Overall advantages of proposed methods are identified and analyzed.

The rest of the paper is organized in eight different sections. Section-2 discusses propagation error problem and categorises transmission errors according to severity. Section-3 proposes two different methods to deal with propagation error problem and introduces these methods. Higher layer protocols overhead and header compression is discussed in section- 4 . In section-5 and section- 6 different propagation environment standardized by 3 GPP for conformance testing are introduced and different bit-error pattern generated by them are discussed. Section-7 discusses different video quality assessment methods including objective and near subjective evaluations. Simulation and results are presented in section-8. Paper concludes in section-9.

\section{RELATED WORK}

Where the transmission error has damaged crucial parts of the bit-streams such as a frame header, the decoder may be unable to decode the frame which it then drops [2]. If this dropped frame is a P-frame, none of the frames that are subsequently coded with reference to this dropped P-frame can be decoded. So in effect all subsequent of frames until the next intra-refresh is dropped. This situation can seriously degrade the received video quality.

If through the use of an upstream message the encoder is made aware of errors in the particular P-frame (P3), the encoder can change the reference frame for the next P-frame $(\mathrm{P} 4)$ to the previous one which was received correctly (P2). P-frames and B-frames after P4 then refer to the correctly decoded P4, rather than the faulty P3 frame. The technique therefore reduces error propagation and frame loss occurring from dropped P-frames.

This method can significantly improve the performance of the received video quality. To implement the NEWPRED feature, both the encoder and decoder need buffer memories for the reference frames. The required buffer memory depends on the strategy of the reference frame selection by the encoder, and transmission delay between the encoder and decoder.

To implement NEWPRED it is important to identify errors at the frame level at the decoding end. Mapping of errors identified by lower layer to the application layer with the precision of a single video frame or video packet often results into complicated process consuming considerable amount of processing capabilities and introduces processing delays. Insertion of CRC bits in the standard MPEG-4 bit-stream at frame level provides simpler solution to this problem [3]. With the insertion of extra bits which are not defined as part of the standard video encoded sequence, would normally result into the incompatible bit-stream with standard decoders. But as mentioned into [3] this would not be the case if these bits are inserted at particular place of the standard MPEG-4 bit-stream. 


\section{INTER-FRAME CODING AND ERROR PROPAGATION}

When a raw video sequence is encoded utilising simple profile at Level 0b of MPEG-4[4], each of the raw video frames is categorized according to the way in which predictive encoding references are used. An Intra-coded (I) frame is coded using information only from itself. A Predictive-coded $(\mathrm{P})$ frame is coded using motion compensated prediction from past reference frame. While a Bidirectionally predictive-coded (B) frame is a frame which is coded using motion and texture compensated prediction from a past and/or future reference frames. A disadvantage of this coding scheme is that, transmission errors occurring in a frame which is used as a reference coding frame for other $\mathrm{P}$ or B frames, causes errors to propagate in the decoded video sequence. This propagation continues until an intra-refresh is applied. Periodic insertion of I-frames can stop this propagation; but as I-frames are entirely intra-coded, they have a minimum amount of data compression.

In wireless environment transmission errors differ significantly depending on the number of factors involved. These errors can occur at any time and with any burst length and hence it is not possible to predict them. At the same time due to random nature of the movements and scenes, the compression achieved in a video clip using MPEG-4 is not uniform throughout the video bit-stream and also not even in the same single video frame. Due to these varying factors it is not predict the exact location of transmission errors but it is possible to broadly categorize them in terms of how destructive they can be in predictive, hierarchical and inter-frame video codec environment. Table-1 categorizes these errors.

Table 1. Error categories

\begin{tabular}{|l|c|}
\hline Error Category & Affected part of the video bit-stream \\
\hline Most critical errors & $\bullet$ Video bit-stream header \\
& $\bullet$ I-frame header \\
& $\bullet$ I-frame data \\
\hline Less critical errors & $\bullet$ P-frame header \\
& $\bullet$ P-frame data \\
\hline Lease critical errors & $\bullet$ B-frame header \\
& $\bullet$ B-frame data \\
\hline
\end{tabular}

As listed in table-1 transmission errors occurring in video bit-stream header, I-frame header and I-frame data can be most destructive and can damage the received video quality at significant degree. As these errors once occurred can propagate into the video bit-stream for considerable time interval and can affect large number of received frames, these are classified as "Most critical errors" to tackle.

"Less critical errors" are classified as errors which occur into P-frame header and P-frame data. Since compare to "most critical errors" the error propagation interval can be smaller in this case these are categorized as comparatively "less critical errors".

Transmission errors occurring in B-frame header and B-frame data affect the received video quality of that particular single B-frame only and do not propagate into video bit-stream as none other received frame is predicatively coded with reference to B-frames. Since this type of errors affects only one single B-frame, these are classified as "Least critical errors".

\section{Proposed Methods TO DEAL With EACH CATEgory OF ERRORS}

This research primarily concentrates on avoiding and then concealing propagation errors as quickly as possible. "Most critical errors" and "Less critical errors" are most likely to affect the received video quality at a significant degree. Thus two different methods are proposed to deal with these errors. The last category of errors that is "Least 
critical errors" are not dealt with in this research since only single frame is affected and transmission error does not propagate. Table-2 lists proposed methods to deal with each category of errors separately.

Table 2. Proposed methods to deal with each category of errors

\begin{tabular}{|l|cl|}
\hline Error Category & \multicolumn{2}{|c|}{ Proposed Method } \\
\hline $\begin{array}{l}\text { Most critical } \\
\text { errors }\end{array}$ & $\bullet$ & $\begin{array}{l}\text { Unequal Error } \\
\text { Protection (UEP). }\end{array}$ \\
\hline Less critical errors & $\bullet$ & NEWPRED \\
\hline $\begin{array}{l}\text { Least critical } \\
\text { errors }\end{array}$ & $\bullet$ & $\begin{array}{l}\text { Are not dealt with in } \\
\text { this research }\end{array}$ \\
\hline
\end{tabular}

These proposed methods are described in following sections.

\subsection{Unequal Error Protection (UEP)}

For video applications Forward Error Correction (FEC) mechanism is suggested in many papers to implement UEP, largely because of the delay introduced by Automatic Repeat Request (ARQ) mechanism between end-toend entities. If ARQ is implemented on air-interface as proposed in a novel UEP mechanism presented here, transmission redundancy can be reduced at a large degree with significantly lower delay involved.

Due to random and bursty nature of errors in wireless environment, if any form of FEC is implemented, it has to be done without any prior knowledge of error location. This can result in significantly large redundancy overhead in bandwidth limited wireless environment.

It is important to develop the UEP scheme which suits the particular communication system and the propagation environment. A generalized UEP scheme can not be applied for all kind of communication systems. Especially where wireless communication systems like UMTS has number of standardized parameters on the different layers of communication protocol stack. Following is the list of limitations of previously proposed methods and requirements for which a specialized UEP mechanism is required for the UMTS.

Due to the bandwidth limitations it is not feasible to have a significant amount of overhead or redundancy as would be the case in most of the FEC implementations. An ARQ mechanism can provide alternative in bandwidth limited environment.

UEP mechanism which relies on the physical layer channel coding does not suit the UMTS implementation. The reason is 3GPP has standardized few Signaling Access Bearer (SABs) and Radio Access Bearer (RABs) and relevant combination of them in 3GPP TS 34.108 [4]. At higher data-rate (more than $32 \mathrm{kpbs}$ ), which is typically required by video applications, not much flexibility is provided to introduce unequal channel coding protection by this standard. So, a UEP mechanism which is compatible with 3GPP standards is required.

When the overhead or redundancy is added to the existing video standard the modified video stream becomes incompatible with the MPEG-4 video standard which can not be decoded by all standard video decoders. With regards to this it is very important that any overhead or redundancy be added in a way which does not actually makes the modified video bit-stream incompatible with the standard.

For above mentioned reasons it is required to propose and investigate a novel UEP mechanism which is designed in accordance with 3GPP and MPEG-4 standards and other requirements. Figure-1 and table-3 proposes such UEP mechanism. 


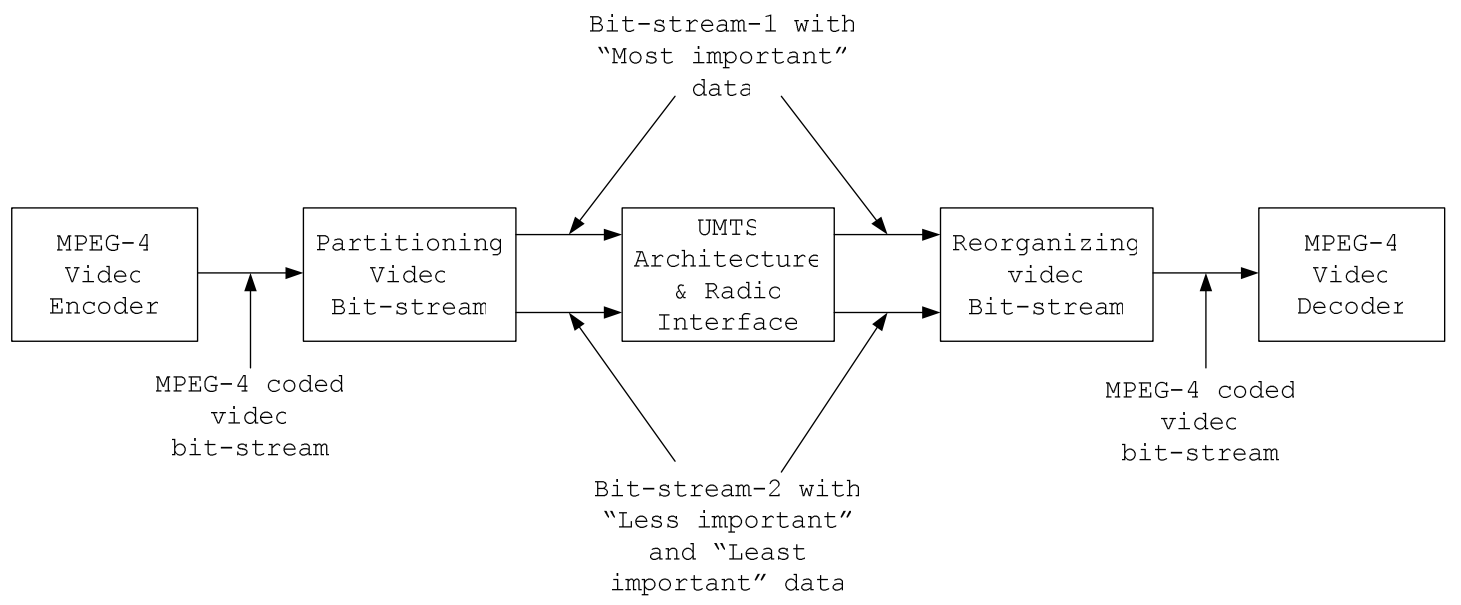

Figure 1. Bit-steam transmission and reception

Table 3. Mapping of data for proposed UEP

\begin{tabular}{|l|l|l|l|}
\hline Data description & Importance of Data & Bit-stream Name & RLC Mode \\
\hline $\begin{array}{l}\text { Video bit-stream header } \\
\text { I-frame header } \\
\text { I-frame data }\end{array}$ & Most important & 1 & AM \\
\hline $\begin{array}{l}\text { P-frame header } \\
\text { P-frame data }\end{array}$ & Less important & 2 & TM \\
\hline $\begin{array}{l}\text { B-frame header } \\
\text { B-frame data }\end{array}$ & Least important & 2 & \\
\hline
\end{tabular}

The transparent mode (TM) data transfer service of UMTS RLC (Radio Link Control) protocol [5] transmits upper layer PDUs without adding any protocol information, possibly including segmentation/reassembly functionality, while the Acknowledged Mode (AM) service transmits upper layer PDUs with guaranteed error-fee delivery to the peer entity using retransmission mechanism at RLC layer. AM mode implemented through a process of error detection at the receiving peer entity and retransmission of the RLC PDU. The advantage of using a retransmission mechanism at the RLC layer instead of transport layer (TCP) is the size of RLC PDUs, which are significantly smaller than TCP segments.

As the MPEG-4 encoded I-frames represent most important data structures as discussed in section-3, in the proposed UEP scheme only I-frames together with video bit-stream header are transmitted using the AM RLC mode, while P and B frames are transmitted using the TM mode. This results in only a small amount of data having to be retransmitted in bursty error-prone environment. In the test scheme, at the application layer the video data is divided into two different bit-streams. Bit-stream-1 contains all I-frames and video bit-stream header while bitstream-2 contains the P and B frames. Bit-stream-1 is transmitted using "Interactive multimedia" ToS class and "Conversational" UMTS QoS class, while bit-stream-2 is transmitted using "Streaming multimedia" ToS class and "Streaming" UMTS QoS class [6].

Because all the I-frame data is transmitted using the AM service, it is expected that all the I-frame PDUs are received without any errors. Those which are received with errors are retransmitted and thus this AQR removes all errors in the received I-frame data. Thus implementation of UEP at RLC layer removed all "Most critical" transmission errors from propagating into video bit-stream. 


\subsection{NEWPRED}

As explained before, propagation error can damage the received video quality significantly. Where the transmission error has damaged crucial parts of the bit-streams such as a frame header, the decoder may be unable to decode the frame which it then drops. If this dropped frame is a P-frame, none of the frames that are subsequently coded with reference to this dropped P-frame can be decoded. So in effect all subsequent frames until the next intra-refresh are dropped. This situation can seriously degrade the received video quality.

The MPEG-4 ISO/IEC 14496 (Part-2) standard provides error robustness and resilience capabilities to allow accessing of image or video information over a wide range of storage and transmission media. Error resilience tools developed for this part of ISO/IEC 14496 can be divided into the three major categories of synchronisation, data recovery and error concealment. The NEWPRED feature falls into the category of error concealment procedures. Where an upstream data channel exists from the decoder to the encoder, NEWPRED or demand intra refresh can be used [5]. NEWPRED is a technique in which the reference frame for inter-frame coding is replaced adaptively according to the upstream messaging from the decoder. The upstream messages indicate which segments are erroneously decoded and on receipt of the upstream message the encoder subsequently will use only the correctly decoded part of the prediction in an inter-frame encoding scheme. In the example shown in figure-2, if through the use of an upstream message the encoder is made aware of errors in the particular P-frame (P3), the encoder can change the reference frame for the next P-frame (P4) to the previous P-frame which was received correctly (P2). P-frames and B-frames after P4 then refer to the correctly decoded P4, rather than the faulty P3 frame. The technique therefore reduces error propagation and frame loss occurring from dropped P-frames. This prevents temporal error propagation without the insertion of intra coded MBs (Macro Blocks) and improves the video quality in the noisy multi-path environments.

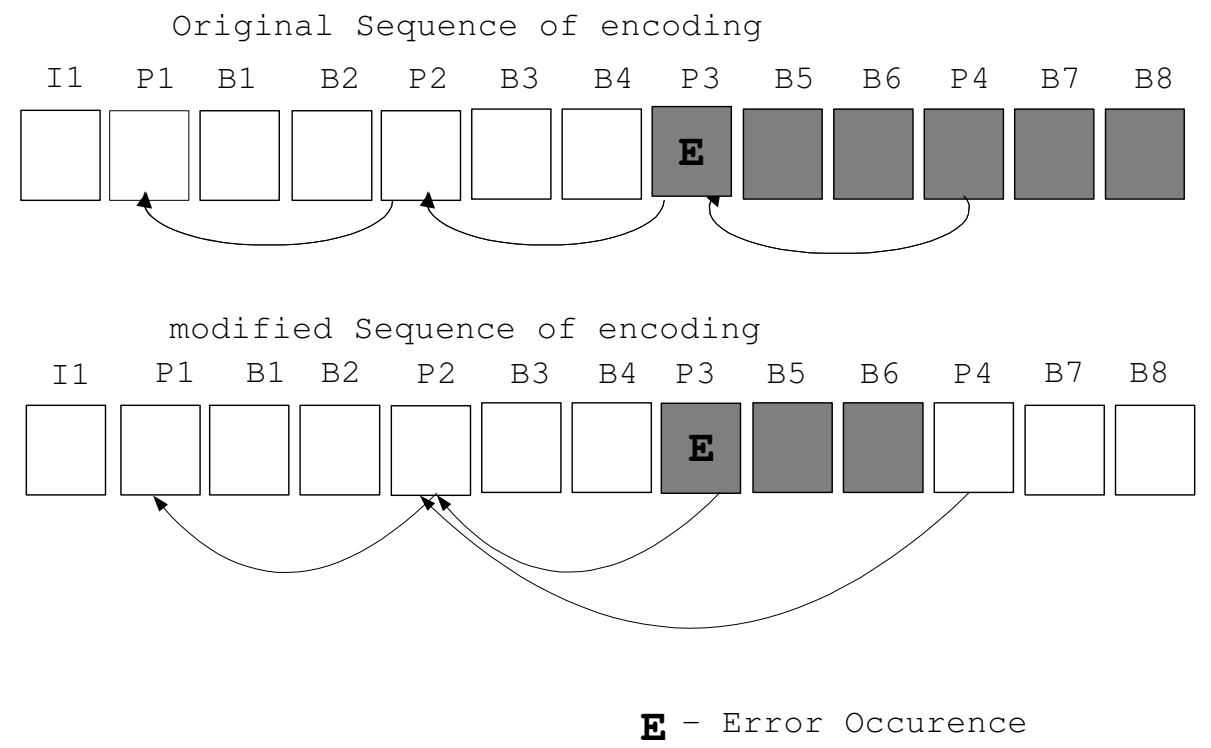

Figure 2. Implementation of NEWPRED

To implement NEWPRED it is important to identify frame level errors at the decoding end. Mapping errors identified by lower layers to the application layer with the precision of a single video frame or video packet is a time consuming and complicated process. Insertion of CRC bits in the standard MPEG-4 bit-stream at the frame level provides a simpler solution to this problem. With the insertion of extra bits which are not defined as part of the standard video encoded sequence [5], would normally result in a bit-stream incompatible with standard 
decoders. However as mentioned into [7] this would not be the case if these bits are inserted at a particular place in the standard MPEG-4 bit-stream. As the decoder is aware of the total number of the macroblocks (MB) in each frame, it starts searching for a new video frame header after decoding these macroblocks. During this process decoder treats the data between the last marcoblock of the frame and the next frame header as padding. If CRC bits are inserted in this part of the bit-stream, then compatibility of the bit-stream with standard MPEG-4 is maintained. In addition, as the inserted CRC is only 16 bits it is not possible for it to emulate any start code sequences of any headers used.

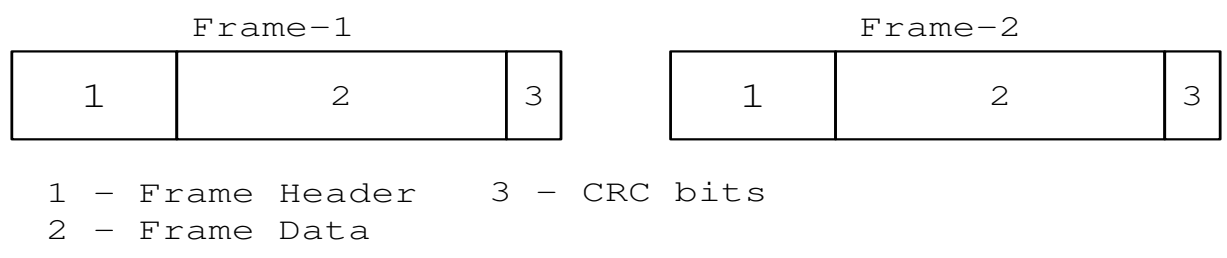

Figure 3. CRC insertion

This method adds extra 16 bits of overhead to each frame but performance improvements in video quality with NEWPRED implementation aided with CRC error detection operation justifies this overhead.

\section{UPPER LAYER PROTOCOL OVERHEADS AND PDCP HEADER COMPRESSION}

UMTS is a complex system where various radio interface protocols are needed to set up, reconfigure and release Radio Bearer (RB) services [8]. Depending upon protocol configuration and the size of the video frames, these headers can be attached to each video frame or multiple video frames can be used as a single payload as defined by RFC-3016. As many successive headers contain a huge amount of redundant data, header compression is applied in the form of Packet Data Convergence Protocol (PDCP) [9]. With PDCP compression, higher layer protocol headers like RTP, UDP and IP headers are compressed into one single PDCP header. In the presented simulation for this paper, header attachments, compression and header removal were achieved using $\mathrm{C}$ programming language. Figure-4 shows the simplified user plane protocols stack between UE (User Equipment) and SRNC (Serving Radio Network Controller).

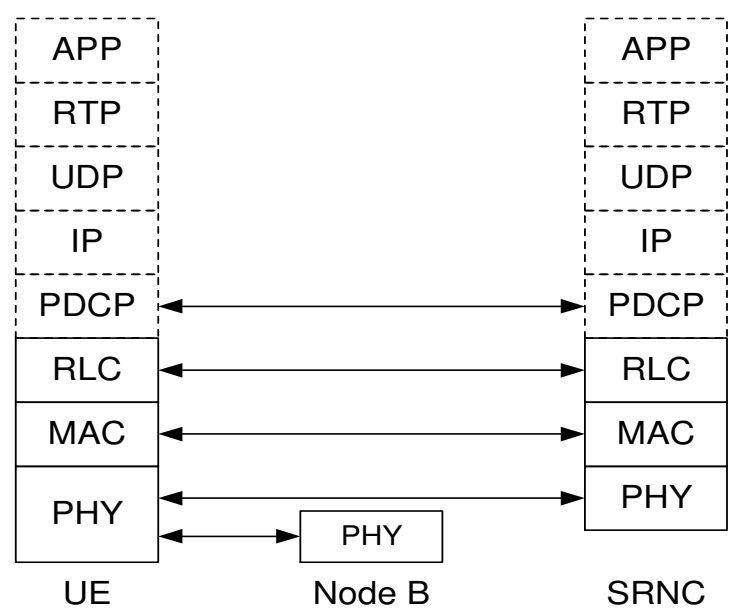

Figure 4. User plan protocol stack between UE and SRNC 
Once the PDCP compression is achieved this packets are then submitted to the RLC layer for the further processing. RLC layer can be configured in any one of the transparent, unacknowledged or acknowledged modes of transmission. RLC then submits the PDU to lower layers, where MAC layer and physical layer procedures are applied as appropriate.

In the presented simulation, MAC layer [10] and physical layer [11] were simulated with SPW 4.2 utility by CoWare to generate link-layer parameters and error patterns for different channel propagation conditions as described below.

\section{GENERATION OF ERROR PATTERNS}

As mentioned, SPW tool by CoWare was used to model an environment to generate error patterns for various types of channel propagation conditions defined by the 3GPP standards [9]. A 64 kbps downlink data channel and 2.5 kbps control channel were used for this UMTS simulation. These two channels were multiplexed and transmitted over the WCDMA air-interface.

The transmission time interval, transmission block size, transmission block set size, CRC attachment, channel coding, rate matching and inter-leaving parameters were configured for both channels in compliance with the specification 3GPP TS 34.108 [1]. The typical parameter set for reference RABs (Radio Access Barriers) and SABs (Signalling Access Barriers) and relevant combinations of them are presented in this standard.

\section{Propagation conditions}

Four different standardized propagation conditions - static, multi-path fading, moving and birth-death were used to generate different error patterns. The typical parameter set for the conformance testing as mentioned in 3GPP TS 25.101 [11] was used for the radio interface configuration. A common set of parameters for all kinds of environment is listed below, while any specific parameters to the environment are mentioned in the respective results sections.

Table 4. Common environment parameters

\begin{tabular}{|l|l|}
\hline Interference & $-60 \mathrm{~dB}$ \\
\hline Received signal / Noise (SNR) & $-3.0 \mathrm{~dB}$ \\
\hline AWGN noise & $4 \times 10-9$ watts \\
\hline Eb/No (Overall) & $6.01 \mathrm{~dB}$ \\
\hline BER (Bit Error Rate) & 0.001 \\
\hline Data Rate (Downlink) & $64 \mathrm{kbps}$ \\
\hline
\end{tabular}

The different propagation conditions produce varying error distribution patterns which lead to the variation in BLER (Block Error Rate) and FER (Frame Error Rate) values shown in the table below.

Table 5. BLER and FER statistic

\begin{tabular}{|l|l|l|}
\hline Channel Propagation Environment & BLER & FER \\
\hline Static propagation condition & 0.031 & 0.0923 \\
\hline Multi-path propagation condition & 0.0065 & 0.0225 \\
\hline Moving propagation condition & 0.036 & 0.088 \\
\hline Birth-death propagation condition & 0.0329 & 0.0851 \\
\hline
\end{tabular}




\subsection{Static propagation condition}

As defined in 3GPP TS 25.101 [11], the propagation for the static performance measurement is Additive White Gaussian Noise (AWGN) environment. No fading and multi-paths exist for this propagation model. The received values of BLER and FER for this propagation condition were 0.031 and 0.0923 respectively.

Figure-5 shows the typical channel behaviour in terms of error occurrences in the interval of 30-bits for the length of 16 seconds.

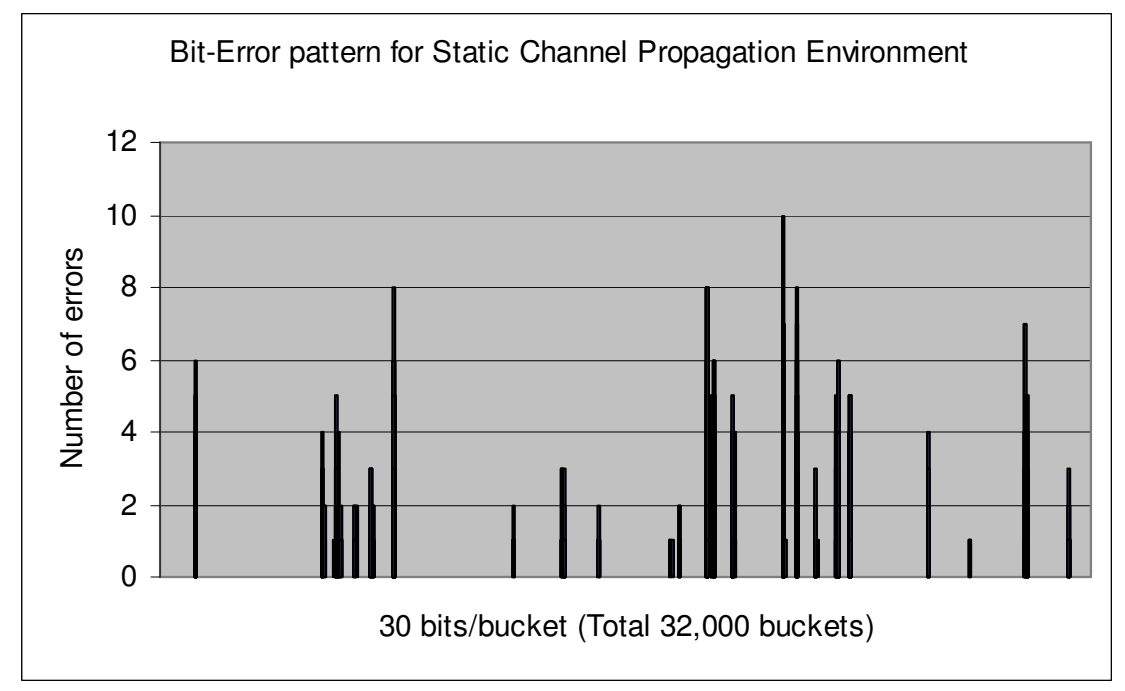

Figure 5. Errors in static channel propagation environment

\subsection{Multi-path fading propagation conditions}

Multi-path fading normally follows Rayleigh fading pattern. In this simulation Case-2 as mentioned by TS $25.101[11]$ is used with the frequency band-1 $(2112.5 \mathrm{MHz})$ and number of paths equals to 3 with relative delay between each path equals to 0,976 and $20000 \mathrm{~ns}$ respectively and relative mean power of $0 \mathrm{~dB}$ for all three paths. The delay model used in this case is fixed. The vehicle speed is configured to be $3 \mathrm{~km} / \mathrm{h}$. The received values of BLER and FER for this propagation condition were 0.0065 and 0.0225 respectively.

Figure-6 shows the typical channel behaviour in terms of error occurrences in the interval of 30-bits for the length of 16 seconds.

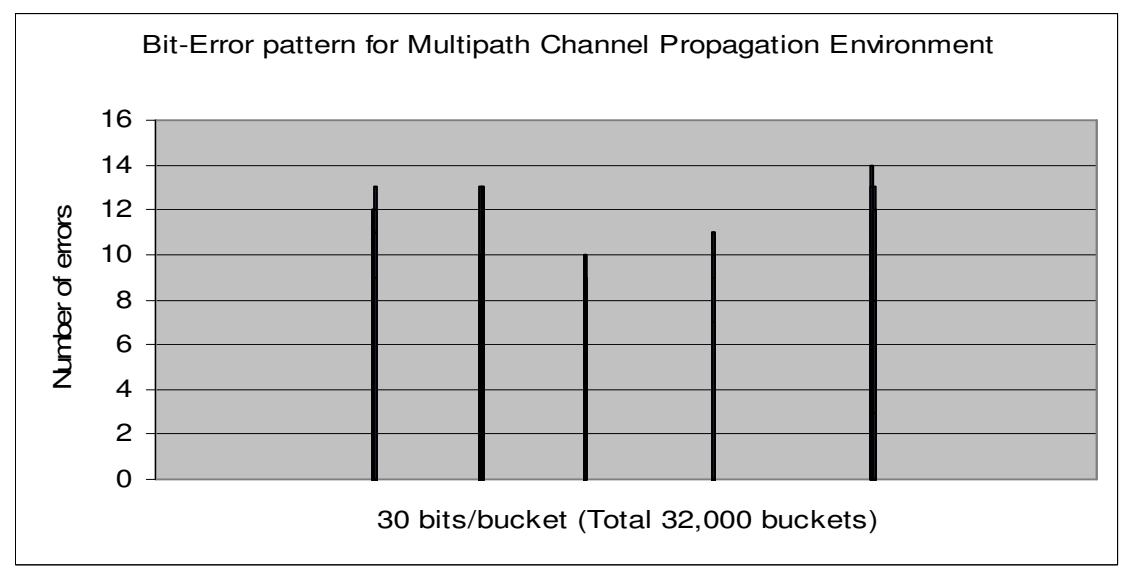

Figure 6. Errors in multipath channel propagation environment 


\subsection{Moving propagation conditions}

The dynamic propagation conditions for this environment for the test of the baseband performance are non fading channel model with two taps as described by 3GPP TS 25.101 [11]. One of the taps is static, Path-0, and other is moving, Path-1. Both taps have equal strengths and phases but unequal time difference between them.

The received values of BLER and FER for this propagation condition were 0.036 and 0.088 respectively.

Figure-7 shows the typical channel behaviour in terms of error occurrences in the interval of 30-bits for the length of 16 seconds.

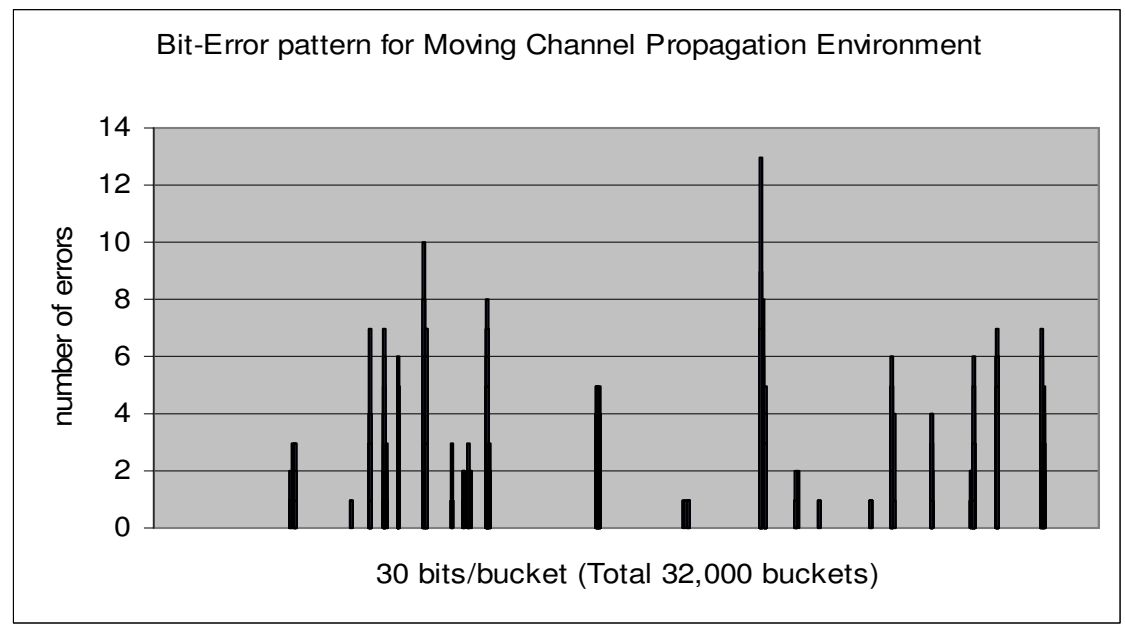

Figure 7. Errors in moving channel propagation environment

\subsection{Birth-Death propagation conditions}

It works similar to the Moving propagation except, in this case both taps are moving. The positions of paths appear randomly and are selected with an equal probability rate. The received values of BLER and FER for this propagation condition were 0.0329 and 0.0851 respectively.

Figure- 8 shows the typical channel behaviour in terms of error occurrences in the interval of 30-bits for the length of 16 seconds.

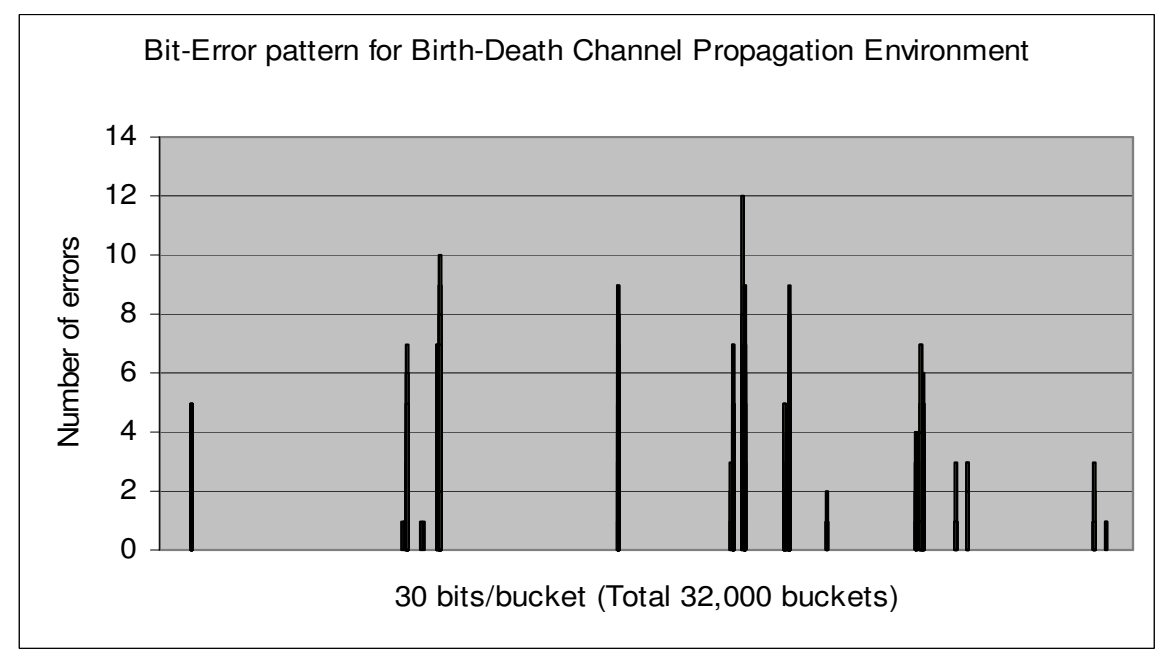

Figure 8. Errors in birth-death channel propagation environment 
It can be seen from BLER and FER numbers for all four different propagation conditions that occurrences of error can differ at a significant degree in different environments.

Generated error patterns are then applied to the data transmitted from the RLC layer. Different RLC modes are simulated using $\mathrm{C}$ programming language.

\section{VIDEO CLIPS AND VIDEO QUALITY ASSESSMENT TECHNIQUES USED}

For the evaluation purpose three standard video test sequences were used, these being: Mother-Daughter, Highway and Foreman. Each of these clips is of 650 frames in length of QCIF $(176 \times 144)$ resolution and is encoded with a standard MPEG-4 codec at $10 \mathrm{fps}$.

The objective video quality is measured by the PSNR (Peak Signal to Noise Ratio) as defined by ANSI T1.801.031996. One more sophisticated model which is developed and tested by ITS (Institute for Telecommunication Sciences) is the Video Quality Metric (VQM) [13] [14]. VQM has been extensively tested on subjective data sets and has significantly proven correlation with subjective assessment. One of the many models developed as a part of this utility is Peak-Signal-to-Noise-Ratio VQM (VQMP). This model is optimized for use on low-bit rate channels and is also used in this paper for the near subjective analysis of the received video sequence.

Typical VQMP is given by:

$$
V Q M p=\frac{1}{1+e^{0.1701 \times(P S N R-25.6675)}}(1)
$$

The higher the PSNR value the better is the objective quality whiles the lower the VQMP value the better is subjective quality of the video sequence.

\section{Simulation Results}

As mentioned before VQMP is used as quality measurement metric in this simulation. Following conventions are used to describe results.

Video clips names:

- $\quad$ Mother and Daughter - MD

- Highway - HW

- Foreman - FM

$V_{Q} M_{P}$ without UEP and without NEWPRED - Results A

$V_{Q} M_{P}$ with UEP and without NEWPRED - Results B

$V_{Q} M_{P}$ without UEP and with NEWPRED - Results C

$V_{Q} M_{P}$ with UEP and with NEWPRED - Results D 
Figure-9 shows PSNR comparison while Table-6 below show $\mathrm{VQM}_{\mathrm{P}}$ scores comparisons for static channel propagation environment.

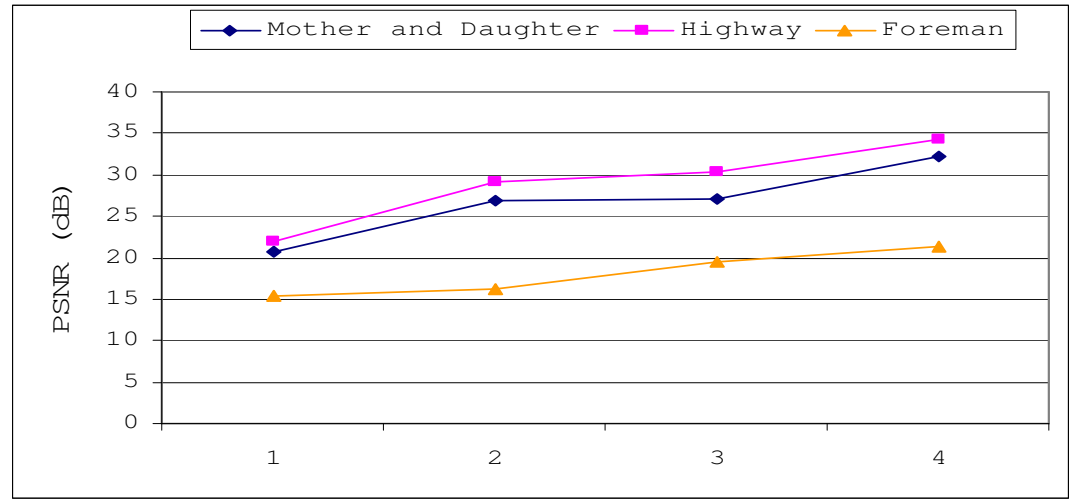

$$
\begin{aligned}
& 1 \text { - Without UEP and without NEWPREL } \\
& 2 \text { - With UEP and without NEWPRED } \\
& 3 \text { - Without UEP and with NEWPREL } \\
& 4 \text { - With UEP and with NEWPRED }
\end{aligned}
$$

Figure 9. PSNR comparisons for static propagation environment

Table 6. VQMP comparisons for static propagation environment

\begin{tabular}{|l|l|l|l|l|}
\hline Video Clip & Results A & Results B & Results C & Results D \\
\hline MD & 0.70 & 0.46 & 0.45 & 0.25 \\
\hline HW & 0.64 & 0.36 & 0.32 & 0.19 \\
\hline FM & 0.85 & 0.83 & 0.74 & 0.68 \\
\hline
\end{tabular}

Figure-10 shows PSNR comparison while Table-7 below show VQMP scores comparisons for multi-path channel propagation environment.

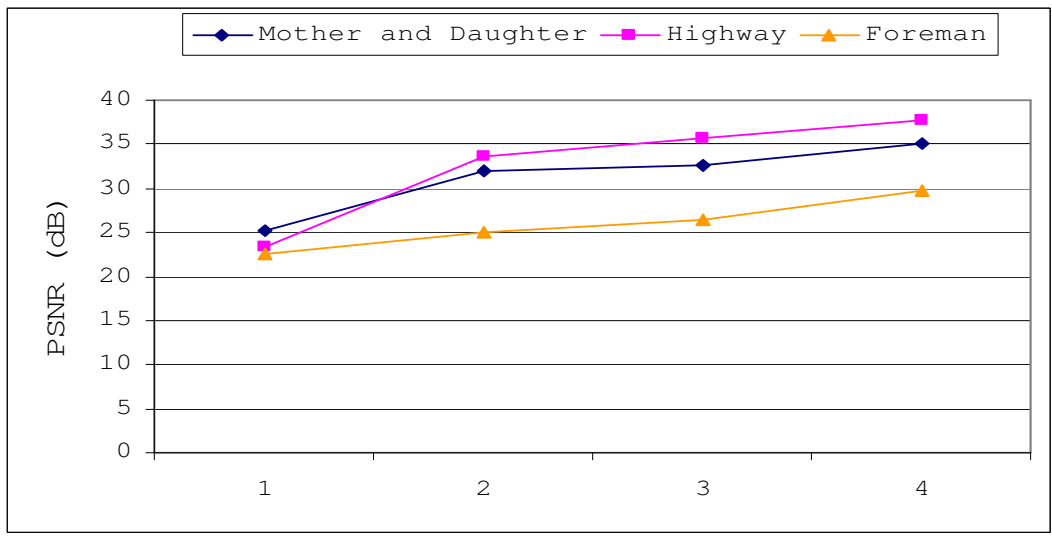

\footnotetext{
1 - Without UEP and without NEWPRED

2 - With UEP and without NEWPREL

3 - Without UEP and with NEWPREL

4 - with UEP and with NEWPRED
}

Figure 10. PSNR comparisons for multi-path propagation environment 
International Journal of Wireless \& Mobile Networks (IJWMN), Vol.2, No.2, May 2010

Table-7: VQMP comparisons for multi-path propagation environment

\begin{tabular}{|l|l|l|l|l|}
\hline \multicolumn{1}{|c|}{ Video Clip } & Results A & \multicolumn{1}{c|}{ Results B } & \multicolumn{1}{c|}{ Results C } & Results D \\
\hline MD & 0.27 & 0.18 & 0.18 & 0.16 \\
\hline HW & 0.37 & 0.21 & 0.18 & 0.13 \\
\hline FM & 0.62 & 0.53 & 0.47 & 0.34 \\
\hline
\end{tabular}

Figure-11 shows PSNR comparison while Table-8 below show VQMP scores comparisons for moving channel propagation environment.

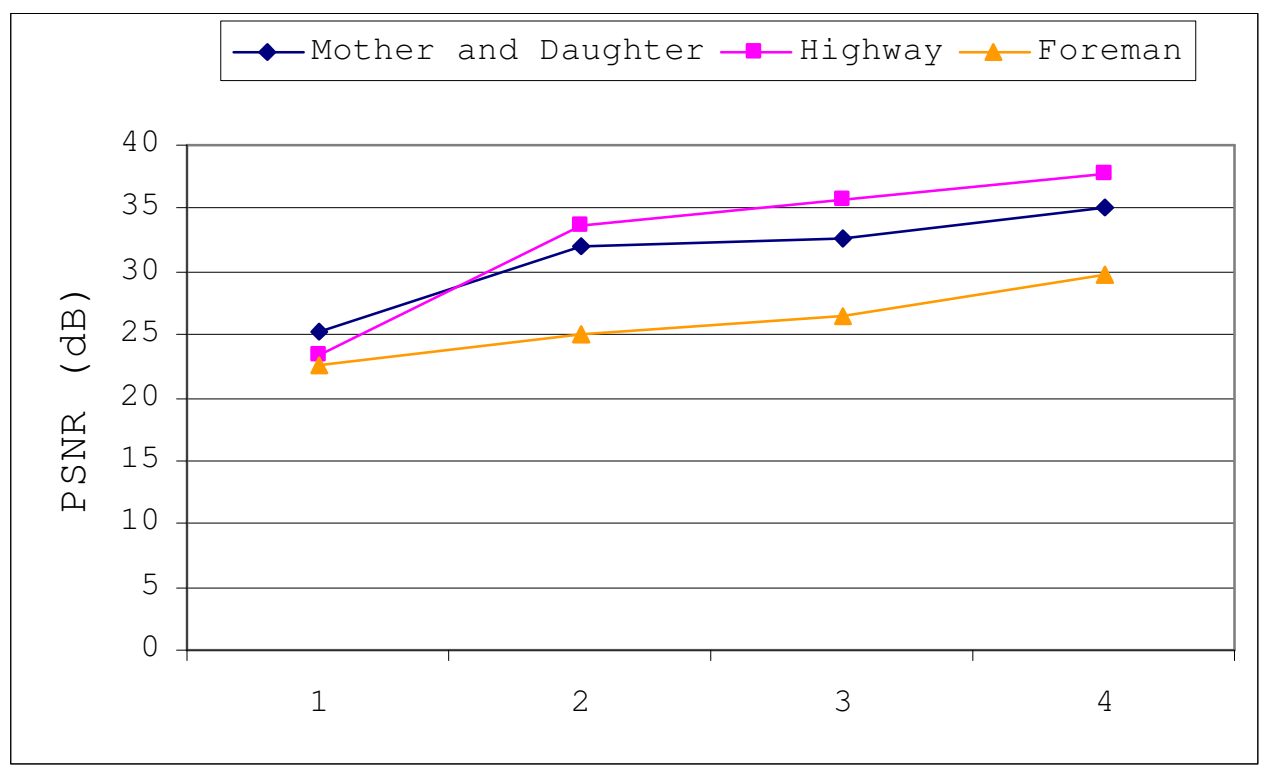

1 - Without UEP and without NEWPREL

2 - With UEP and without NEWPRED

3 - Without UEP and with NEWPRED

4 - With UEP and with NEWPRED

Figure 11. PSNR comparisons for moving propagation environment

Table 8. VQMP comparisons for moving propagation environment

\begin{tabular}{|l|l|l|l|l|}
\hline Video Clip & Results A & Results B & Results C & Results D \\
\hline MD & 0.63 & 0.45 & 0.44 & 0.23 \\
\hline HW & 0.55 & 0.35 & 0.31 & 0.26 \\
\hline FM & 0.83 & 0.70 & 0.67 & 0.52 \\
\hline
\end{tabular}


Figure-12 shows PSNR comparison while Table-9 below show VQMP scores comparisons for birth-death channel propagation environment.

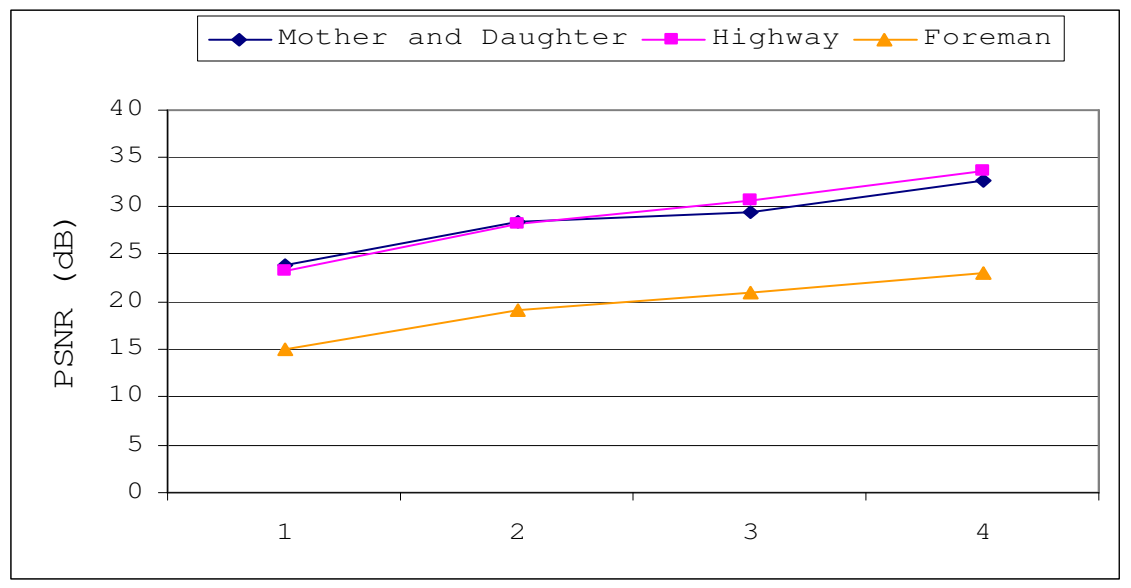

$$
\begin{aligned}
& 1 \text { - Without UEP and without NEWPREL } \\
& 2 \text { - With UEP and without NEWPRED } \\
& 3 \text { - Without UEP and with NEWPRED } \\
& 4 \text { - With UEP and with NEWPRED }
\end{aligned}
$$

Figure 12. PSNR comparisons for birth-death propagation environment

Table 9. VQMP comparisons for birth-death propagation environment

\begin{tabular}{|l|l|l|l|l|}
\hline Video Clip & Results A & Results B & Results C & Results D \\
\hline MD & 0.58 & 0.32 & 0.35 & 0.27 \\
\hline HW & 0.49 & 0.40 & 0.31 & 0.22 \\
\hline FM & 0.85 & 0.72 & 0.68 & 0.45 \\
\hline
\end{tabular}

The received VQMP values for all video sequences are improved by implementation of UEP and NEPWRED methods.

It can be observed that in most cases the VQMP values show greater improvement with implementation of the NEWPRED method than the improvements obtained with the UEP method. Errors in the wireless environment occur in bursts and are random in nature. Due to the time varying nature of the wireless environment it is not possible to predict the exact location of the error. The NEWPRED method provides protection to the P-frames while UEP is aimed at protecting the I-frames. As P-frames are much more frequent than I-frames in the encoded video sequence, P-frames are more susceptible to these bursty randomly distributed errors. This explains why the NEWPRED implementation gives better performance than the UEP implementation.

Combined implementation of UEP and NEWPRED methods always outperforms single method implementation and gives the best quality of received video. The same observation is made in terms of performance assessment using the VQMP score in most cases.

Objective assessment of VQMP scores is highly dependent on bit-by-bit comparison which does not take into consideration the number of frozen frames. In most cases frozen frames give better correlation compared to frames with propagation errors. 


\section{CONClusion}

As can be seen from the simulation results, implementation of UEP and NEWPRED results in significant improvements on the received video quality. UEP does not introduce a great deal of forward error correction but improvement achieved using this method is significantly higher. NEWPRED adds additional processing overhead but again both subjective and objective video quality improvements observed in all channel environments is significant. As can be seen from the simulation results, implementation of UEP and NEWPRED results in significant improvements on the received video quality. Improvements achieved using these methods provides extra margin for network operators to increase capacity. With implementation of these error concealment methods the same video quality can be achieved using a lower Eb/No which in turn provides flexibility for network operator to increase the number of users.

\section{REFERENCES}

[1]. Pereira, F., Ebrahimi, T., The MPEG-4 Book; Prentice Hall PTR (July 20, 2002), ISBN-10: 0130616214.

[2]. C.Kodikara, S.Worrall, S. N. Fabri, A.M. Kondoz. 'Performance Evaluation of MPEG-4 video telephony over UMTS', IEE 4th International Conference on 3G Mobile Communication Technologies (3G 2003), IEE Conference Publication N0. 494, London, UK, 25-27 June 2003, pp-73-77.

[3]. S.T. Worrall, A.H. Sadka, P. Sweeney and A.M. Kondoz.: 'Backward compatible user defined data insertion into MPEG-4 bitstreams', IEE Electronic Letters, 8th June 2000, Vol. 36, No.12, pp. 1036.

[4]. International Standard ISO/IEC 14496-2: Information Technology - Coding of audio-visual objects-Part 2, Visual, International Organisation for Standardization. 2001.

[5]. 3GPP, Technical Specification Group Radio Access Network; Radio Link Control (RLC) protocol specification; 3GPP TS 25.322, V4.12.0.

[6]. 3GPP, Technical Specification Group Services and System Aspects; Quality of Service (QoS) concept and architecture, 3GPP TS 23.107 v4.0.0.

[7]. Worrall, S., Sadka, A., Sweeney, P., Kondoz, A., Backward compatible user defined data insertion into MPEG-4 bitstream. IEE Electronics letters.

[8]. 3GPP, Technical Specification Group Radio Access Network; Radio interface protocol architecture; 3GPP TS 25.301 (2002-09), Ver 5.2.0.

[9]. 3GPP, Technical Specification Group Radio Access Network; PDCP protocol specification; 3GPP TS 25.331 (200312), Ver 6.0.0.

[10]. 3GPP, Technical Specification Group Radio Access Network; MAC protocol Specification; 3GPP TS 25.321, V4.0.0.

[11]. 3GPP, Technical Specification Group Radio Access Network; UE radio transmission and reception (FDD); 3GPP TS 25.101 (2003-12), Ver 6.3.0.

[12]. 3GPP, Technical Specification Group Terminals; Common test environment for UE conformance testing; 3GPP TS 34.108 (2003-12), Ver 4.9.0.

[13]. ATIS Technical Report T1.TR.74-2201: Objective Video Quality Measurement using a Peak-Signal-to-Noise Ratio (PSNR) Full Reference Technique. October 2001., Alliance for Telecommunications Industry Solutions.

[14]. Wolf, S., Pinson, M., Video Quality Measurement Techniques. June 2002, ITS, NTIA Report 02-392.

\section{Authors}

Dr. Bhumin Pathak received his M.Sc. and Ph.D. degree from Oxford Brookes University, Oxford, UK. He has been working as Systems Engineer at Airvana Inc., since March 2007.

Dr. Geoff Childs is Principal Lecturer at School of Technology at Oxford Brookes University, Oxford, UK.

Dr. Maaruf Ali is Senior Lecturer at School of Technology at Oxford Brookes University, Oxford, UK. 